Hans Becker, Helmut Däuker, Anja Guck-Nigrelli, Lily Gramatikov, Parfen Laszig, Helmut Lüdeke, Gerhard Schneider, Matthias Richter, Sabine Schluckwerder \& Rolf Vogt

\title{
Zur Gründungsgeschichte der Zeitschrift
}

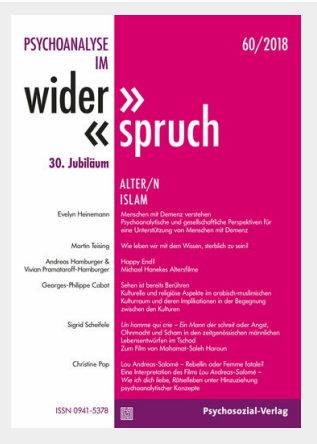

Psychoanalyse im Widerspruch

30. Jahrgang, Nr. 2, 2018, Seite 9-11

Psychosozial-Verlag

DOI: $10.30820 / 8241.02$ 


\section{Impressum}

\section{Psychoanalyse im Widerspruch}

ISSN 0941-5378

Herausgeber:

Institut für Psychoanalyse und Psychotherapie Heidelberg-Mannheim (IPP) und Heidelberger Institut für Tiefenpsychologie (HIT)

\section{Redaktion:}

Hans Becker, Helmut Däuker, Anja GuckNigrelli, Lily Gramatikov, Parfen Laszig, Helmut Lüdeke, Gerhard Schneider, Matthias Richter, Sabine Schluckwerder, Rolf Vogt

Leitender Redakteur:

Parfen Laszig

redaktion@parfen-laszig.de

Redaktionsadresse:

Institut für Psychoanalyse und Psychotherapie

Heidelberg-Mannheim

Alte Bergheimerstraße 5

D-69115 Heidelberg

Telefon und Telefax: 062 21/18 4345

Manuskripte:

Die Redaktion lädt zur Einsendung von Manuskripten ein. Mit der Annahme des Manuskriptes erwirbt der Verlag das ausschließliche Verlagsrecht auch für etwaige spätere Veröffentlichungen.

Verlag:

Psychosozial-Verlag

Walltorstraße $10 \cdot 35390$ Gießen

Telefon: 06 41/96 997826

Telefax: 06 41/96997819

bestellung@psychosozial-verlag.de
Bezug:

Für das Jahresabonnement EUR 29,90 (inkl. MwSt.) zuzüglich Versandkosten. Studentenabonnement 25\% Rabatt (inkl. MwSt.) zuzüglich Versandkosten. Lieferungen ins Ausland zuzüglich Mehrporto. Das Abonnement verlängert sich jeweils um ein Jahr, sofern nicht eine Abbestellung bis acht Wochen vor Beendigung des Bezugszeitraums erfolgt. Preis des Einzelheftes EUR 19,90.

Bestellungen von Abonnements bitte an den Verlag, bestellung@psychosozial-verlag.de, Einzelbestellung beim Verlag oder über den Buchhandel.

Anzeigen:

anzeigen@psychosozial-verlag.de

Erscheinungsweise: Zweimal im Jahr.

Copyright:

(C) 2018 Psychosozial-Verlag, Gießen

Die in der Zeitschrift veröffentlichten Beiträge sind urheberrechtlich geschützt. Alle Rechte, insbesondere das der Übersetzung in fremde Sprachen, bleiben vorbehalten. Kein Teil dieser Zeitschrift darf ohne schriftliche Genehmigung des Verlages in irgendeiner Form durch Fotokopie, Mikrofilm oder andere Verfahren - reproduziert oder unter Verwendung elektronischer Systeme verarbeitet, vervielfältigt oder verbreitet werden. Fotokopien für den persönlichen und sonstigen eigenen Gebrauch dürfen nur von einzelnen Beiträgen oder Teilen daraus als Einzelkopien hergestellt werden.

Satz:

metiTec-Software, me-ti GmbH, Berlin 


\section{Zur Gründungsgeschichte der Zeitschrift}

Psychoanalyse im Widerspruch Nr. 60, 30 (2) 2018, 9-11

https://doi.org/10.30820/8241.02

www.psychosozial-verlag.de/piwi

1988, bei der Idee, eine psychoanalytische Zeitschrift des Heidelberger Instituts für Psychoanalyse und Psychotherapie Heidelberg-Mannheim e. V. zu initiieren, kam es zunächst zu heftigen Auseinandersetzungen. Eine Gruppe bevorzugte die Idee eines institutsinternen Informationsblattes, durchgesetzt hat sich dann das Vorhaben einer überregionalen Zeitschrift. Das inhaltliche Konzept sollte der zunehmenden Medizinalisierung der Psychoanalyse entgegenwirken, der Potenz der Psychoanalyse als Kulturtheorie, als Möglichkeit der Selbstreflexion und der Analyse hierarchischer Strukturen in Politik und Gesellschaft, aber auch gerade in den Ausbildungsinstituten eine Form geben.

Die Zeitschrift hatte zunächst zwei externe gesellschaftskritische Geburtshelferinnen. Zum einen die Karikaturistin Marie Marcks, die das erste Cover entwarf.

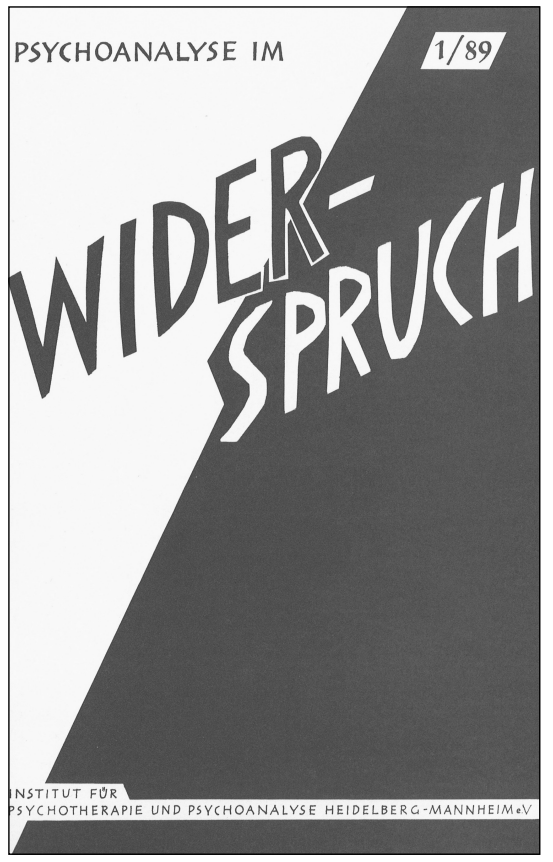


Die andere war Marianne von Eckardt, die unter anderem gemeinsam mit Alexander Mitscherlich den ersten internationalen psychoanalytischen Nachkriegskongress mit deutscher Beteiligung in Amsterdam besuchte. Beide Eltern von Frau von Eckardt waren um die Jahrhundertwende politisch aktiv: die Mutter, Else von Richthofen, war eine der ersten Frauenrechtlerinnen in Deutschland und die Lebensgefährtin von Max Weber, der Vater war Finanzminister in der Räterepublik unter dem ersten bayerischen Ministerpräsidenten Kurt Eisner. Als Übersetzerin von Herbert Marcuses »Triebstruktur und Gesellschaft« hatte Marianne von Eckardt die Idee, die Zeitschrift » Widerspruch « zu nennen.

Ein letzter Versuch der Verhinderung kam vom damaligen Lehrstuhlinhaber, der in einer Mitgliederversammlung den Antrag stellte, dass sich die Zeitschrift ökonomisch selbst tragen müsse. Der ersten Redaktion gehörten Hans Becker, Manfred Drücke, Werner Knauss, Jürgen Krambeck, Joachim Küchenhoff, Helmut Lüdeke, Karl Metzner, Hildegard Parekh, Gerhard Schneider und Wolfgang Senf an.

Der Anpassungsprozess an den Zeitgeist hatte schon damals dazu geführt, dass die Psychoanalyse in der Medizin kaum noch Bedeutung hatte. Schwerpunkt ist heute der Bereich der Kultur- und der Sozialwissenschaften. Hier erlebt die Psychoanalyse geradezu einen Boom.

Die Zeitschrift entstand auch aus der von Hans Becker mitinitiierten, seit weit über 30 Jahren bis heute bestehenden Arbeitsgruppe »Psychoanalyse und Politik «. Diese Gruppe hatte damals den lange anstehenden Generationswechsel im Institut angestoßen. So ist es kein Zufall, dass Mitglieder der Arbeitsgruppe, wie Manfred Drücke, Werner Knauss und Helmut Lüdeke in der Redaktion vertreten waren. Mit Hildegard Parekh, Alexandre Métraux und jetzt Parfen Laszig, die die Redaktionsleitung übernommen hatten, war die Existenzsicherung der Zeitschrift in besten Händen.

In den letzten 30 Jahren gab es immer wieder ermutigende und entmutigende Episoden. Auf dem Höhepunkt der politischen Auseinandersetzung in der Redaktion der Psyche, bei der es um den Ausschluss der zu gesellschaftskritisch denkenden Gruppe um Helmut Dahmer ging, zog beispielsweise Paul Parin eine dort eingereichte Publikation aus der Psyche zurück und übergab sie bewusst an unsere Zeitschrift. Parin hatte immer wieder vertreten, dass in einer Analyse nicht nur die Abwehr, sondern auch die unbewussten Anpassungsmechanismen Berücksichtigung finden müssen - ein Aspekt, der in unserer Zeitschrift immer einen hohen Stellenwert hatte.

Mehrere Jahre nach Erscheinen unserer Zeitschrift kam es zu einem legendären Treffen in Frankfurt. Von Hellmut Becker, dem Leiter des Max- 
Planck-Instituts für Bildungsforschung in Berlin, Horst Eberhard Richter und - später bei einem Treffen in Heidelberg - Hans-Jürgen Wirth wurde die Idee entwickelt, die Zeitschriften psychosozial und Psychoanalyse im Widerspruch zu fusionieren. Obwohl wir die Zeitschrift psychosozial sehr schätzten, nahmen wir von der Idee Abstand. Wir befürchteten unsere Unabhängigkeit zu verlieren, von der Abonnentenzahl abhängig zu werden, was durchaus Einfluss auf den Inhalt hätte nehmen können. Wie man heute sehen kann, ist diese Angst verflogen.

Die Verbreitung der Zeitschrift ging nicht vorwiegend über die Zahl der Abonnenten, sondern über die Bibliotheken der Institute und Lehrstühle. Wir mussten feststellen, dass mit der Veränderung der Bedeutung der Psychoanalyse, v. a. auch an den Lehrstühlen, die Bibliotheksexemplare abbestellt wurden. Mit dem Lehrstuhlwechsel, z. B. in Heidelberg, war die Zeitschrift sehr schnell eliminiert.

Unserem Verleger Hans-Jürgen Wirth danken wir für sein inhaltlich sehr liebevolles Geleitwort und für seine zuverlässige Unterstützung für eine ökonomisch nicht allzu erfolgreiche Zeitschrift.

Wir hoffen, wir können unser inhaltliches Vorhaben, Querdenkern und auch Außenseitern ein Forum zu geben, aufrechterhalten. Gerade aus dieser Gruppe kommen erfahrungsgemäß die innovativsten Ideen.

Die Redaktion 